\title{
Detection of manufacturing defects in ceramic matrix composite (CMCs) by using the active thermography
}

\author{
by L. Gavérina*, A. Debarre*, F. Passilly* and J.-M. Roche*
}

* ONERA, Materials and Structures Department, Châtillon, 92320, France, ludovic.gaverina@onera.fr

\begin{abstract}
Defects such as inhomogeneities or cracks are often present after elaboration of composites materials. In this paper, a Non Destructive Testing approach based on active infrared thermography is used to investigate porosity of oxide/oxide CMC specimens. In this study, pulsed thermography in reflection or transmission modes is used in order to correlate microstructural defects and mechanical properties.
\end{abstract}

\section{Introduction}

An increase in service temperatures is required to improve the thrust-to-weight ratio for high performance aeroengines so the implementation of ceramic matrix composite (CMCs) is considered. During the elaboration process of an oxide based composite, a micro-porosity network is introduced. The homogeneity of the network and the absence of larger pores is a key factor for the mechanical properties of the material. A pore is defined as an inclusion of air and the porosity rate is defined as the volume of pores compared to the volume of the material. Destructive technics such as observing slices with Scanning Electronic Microscopy or mercury porosimetriy can't be used on reals parts. None destructive methods (ultrasonic testing, X-ray tomography...) have been used to characterize this composite. In this work, pulsed IR thermography which is a conventional non-destructive testing approach that offers many advantages (a rapid measurement, simple set-up...) has been proposed. A porosity map and an estimation of the porosity rate by correlating the temperature and the porosity distribution has been obtained [1]. The objective is now to go further and analyze the presence of macropores that are defined as a pore with a size of 10-100 $\mu \mathrm{m}$.

\section{Detection of porosity}

In the latest investigations which had been made [1], two halogen lamps whit $6 \mathrm{~kJ}$ each are used to provide the thermal solicitation to the sample. The surface of the specimen is preliminary painted to have an emissivity close to 0.97 . The IR camera which was used during experiments is a FLIR X6540sc MWIR camera $(640 \times 512$ pixels, NETD $20 \mathrm{mK}$, pitch $15 \mu \mathrm{m}$, spectral band $1.5-5 \mu \mathrm{m}$ ) and frame rate of the camera was $150 \mathrm{~Hz}$.

Figure.1.a shows the normalized thermogram of rear face of the oxide/oxide CMC specimen submitted to a flash heating. The presence of the defects is clearly seen. The sound area is identified in blue color while the damaged one is in red color.

Figure.1.b and .c shows the second order derivative has been applied to TSR images [2]. The defects on the surface of the specimen among the porosity inside the specimen are revealed. In Figure.1.b, the defects appear directly in the second order derivative image. It is means that the defect is at the surface and shoud be some lack of matter.

a)

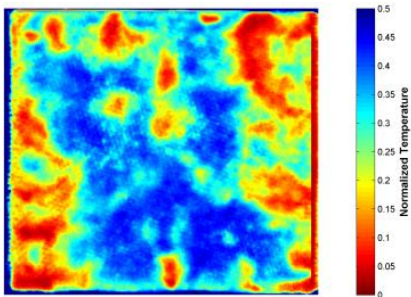

b)

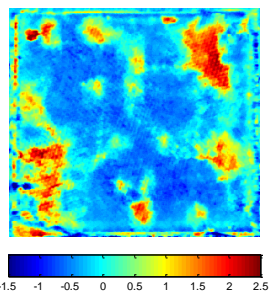

c)

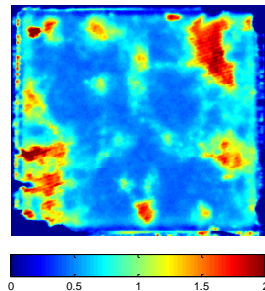

Fig. 1. (a) Rear-face thermograms at optimal time (0.19s); front-face $2^{\text {nd }}$ logarithmic TSR thermograms : (b) at early time $(0,05 \mathrm{~s})$ and (c) late time (2 s).

\section{Mechanical testing}

4-point bending tests have performed using the set up described in Figure 2 in order to characterize the mechanical behavior of this ceramic matrix composite. 

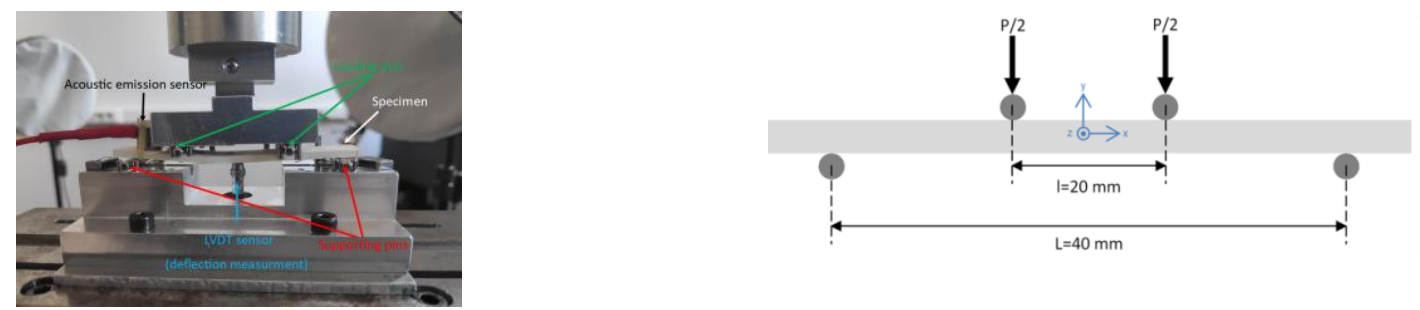

Fig. 2. Four-point bending set up

At the center of the specimen, analytical relationships can used to calculate the maximum stress and strain [3]:

$$
\sigma=\frac{3 P(L-l)}{2 b e^{2}}=\frac{3 P l}{2 b e^{2}} ; \epsilon=\frac{12 e f}{3 L^{2}-(L-l)^{2}}=\frac{12 e f}{11 l^{2}}
$$

with $\sigma$ the applied stress $(M P a), P$ the load $(N), \epsilon$ the strain, $f$ the deflection $(m m), L$ and $l$ the spacing between supporting pins and loading pins $(\mathrm{mm}), b$ the width of the specimen $(\mathrm{mm})$ and $e$ its thickness $(\mathrm{mm})$.

A strain field can be obtained using Digital Image Correlation (DIC). An example is shown in the Figure 4. The specimen is undeformed (Figure 3.a), deformed (Figure 3.b and c) and broken (Figure 3.d)

a)

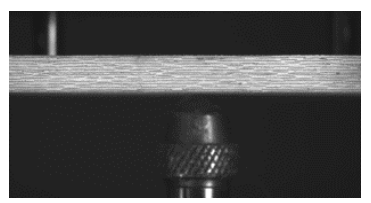

b)

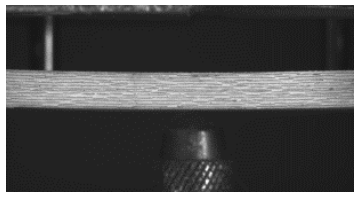

c)

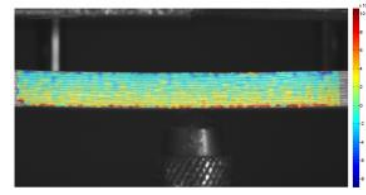

d)

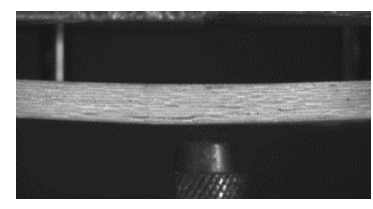

Fig. 3. Four-point flexural test: (a) undeformed specimen, (b) deformed specimen, (c) deformed specimen with strain calculation and $(d)$ delaminating specimen

Tests have been carried out on sample chosen for being considered as healthy or having defects. Since the stress is higher near defects, healthy sample are expected to have a higher strength (breaking stress). Unfortunately no correlation has been found between NDT and mechanical results. The defects detected are not the ones that matter for the understanding of the mechanical behavior.

After having determined a porosity map of the material, it was important to locate the macropores to be able to choose where representative specimen can be machined. Since the technic is also dependent of surface defects such as lack of matrix, it is difficult to locate the pores in the thickness. Using a FLIR X6901sc sls LWIR camera allows a higher sampling frequency (Figure 5) and a better characterization of the drop on the thermograms.

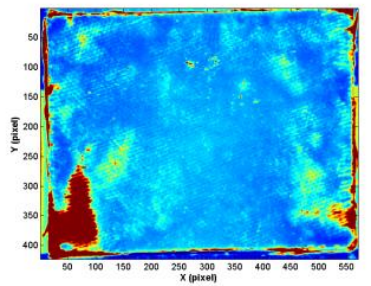

Fig. 5. Averaged thermogram over several hundreds of the front face of the specimen with a frame rate of the camera at $1000 \mathrm{~Hz}$.

\section{REFERENCES}

[1] Gaverina L, Debarre A, Trottier C, Passilly F, Roche J.M. Comparative study of Pulsed IR Thermography and Ultrasonic Testing to obtain a porosity mapping of oxide/oxide CMC specimens. In QIRT Asia Tokyo 2019.

[2] Roche, Jean-Michel, and Daniel L. Balageas. "Common tools for quantitative pulse and step-heating thermography-part II: experimental investigation." Quantitative InfraRed Thermography Journal 12.1 (2015): 123

[3] S. P. Timoshenko and J. N. Goodier, "Theory of Elasticity," 3rd Edition, McGraw Hill, New York, 1970 